TRANSACTIONS OF THE

AMERICAN MATHEMATICAL SOCIETY

Volume 349, Number 4, April 1997, Pages 1487-1502

S 0002-9947(97)01692-9

\title{
ON THE HOMOTOPY TYPE OF $B G$ FOR CERTAIN FINITE 2-GROUPS $G$
}

\author{
CARLOS BROTO AND RAN LEVI
}

\begin{abstract}
We consider the homotopy type of classifying spaces $B G$, where $G$ is a finite $p$-group, and we study the question whether or not the $\bmod p$ cohomology of $B G$, as an algebra over the Steenrod algebra together with the associated Bockstein spectral sequence, determine the homotopy type of $B G$. This article is devoted to producing some families of finite 2-groups where cohomological information determines the homotopy type of $B G$.
\end{abstract}

\section{INTRODUCTION}

Let $G$ and $H$ be finite $p$-groups, which agree on some of their cohomological invariants. The question whether $G$ and $H$ are isomorphic groups has a negative answer in general. For instance, mod-2 cohomology algebra cannot differ between dihedral 2-groups of various orders, even if the action of the Steenrod algebra is taken into account. A similar fact is known for various metacyclic 2-groups, among which one counts the quaternion and semidihedral groups [15]. In a recent paper, I. Leary exhibited examples of distinct 3-groups having isomorphic integral cohomology rings [12]. Further examples of distinct finite 2-groups, which mod-2 cohomology algebra cannot tell apart, are given in [16]. However if one requires, in a sense that will be made precise later, that the given cohomological data on $G$ includes its Bockstein spectral sequence then, although we are not able to prove the contrary, we are not aware of an example of two distinct $p$-groups which cohomology cannot tell apart.

The question becomes significantly more complicated when one considers a finite $p$-group $G$ and a $p$-complete space $X$ such that $B G$ and $X$ have the same cohomological invariants and asks whether $X$ and $B G$ have the same homotopy type. This last question is reduced to the previous one if one can show for a particular example that $X$ has the homotopy type of $B H$ for "some" finite $p$-group $H$. One might thus wonder to what extent does cohomological data determine isomorphism type for finite $p$-groups $G$, or more generally, the homotopy type of $B G$.

In this note we shall mainly be interested in the second question. We restrict attention to the prime 2 and study some examples of finite 2-groups $G$, where the

Received by the editors May 19, 1995.

1991 Mathematics Subject Classification. Primary 55R35; Secondary 55R40, 55Q52.

Key words and phrases. Classifying spaces, finite 2-groups, cohomology, Steenrod squares, Bockstein spectral sequence.

C. Broto is partially supported by DGICYT grant PB94-0725.

R. Levi is supported by a DFG grant. 
homotopy type of $B G$ is determined by its cohomology. Further examples where the results are not as conclusive are also given.

Unless otherwise specified all spaces are assumed to have the homotopy type of a 2-complete $\mathrm{CW}$ complex. The cohomology of a space is considered as an object in the category $\mathcal{K}_{\beta}$. It is, roughly speaking, the category of unstable algebras over the Steenrod algebra, in which the higher Bockstein operators are being taken into account. It is immediate, for instance, that every finite abelian 2-group $G$ gives rise to a unique object of $\mathcal{K}_{\beta}$, characterizing $B G$ up to homotopy and hence the group $G$, up to isomorphism. We say that two spaces $X$ and $Y$ are comparable if $X$ and $Y$ give rise to isomorphic objects in $\mathcal{K}_{\beta}$. We say that the homotopy type of a space $X$ is determined by its cohomology if any space $Y$, comparable to $X$, is homotopy equivalent to it.

The first family of groups we consider is the dihedral 2-groups.

Theorem 1.1. Let $D_{2^{n}}$ denote the dihedral group of order $2^{n}$. Then the homotopy type of $B D_{2^{n}}$ is determined by its cohomology.

A considerably larger family of 2-groups, containing the dihedral and quaternion groups of order 8 , are the extra special 2-groups which we consider next.

Theorem 1.2. Let $G$ denote an extra special 2-group. Then the homotopy type of $B G$ is determined by its cohomology. In fact in almost all cases, the homotopy type of $B G$ for an extra special 2-group $G$ is determined by the cohomology algebra of $B G$.

The examples we present next appear less favorable. Indeed a basic question in the subject is whether in general cohomology forces at least the fundamental group of a space $X$, given that $X$ is comparable to $B G$ for some finite $p$-group $G$. In the following examples we were not able to determine a complete answer to this question. Thus one might suspect that an example of a space $X$ comparable to $B G$ yet not homotopy equivalent to it might arise from studying the groups $G$ considered below.

Proposition 1.3. Let $G=Q_{2^{n}}$ denote the generalized quaternion group of order $2^{n}$. Then for $n \leq 5$ the homotopy type of $B G$ is determined by its cohomology. For $n>5$ let $X$ be a space comparable to $B G$. Then either $\pi_{1} X \cong Q_{2^{k}}$ for some $4 \leq k \leq n-2$ or $X \simeq B G$.

The question of whether or not the homotopy type of $B Q_{2^{n}}$ is determined by its cohomology for $n>5$ is reduced in section 5 below to understanding free actions of the group $Q_{16}$ on certain simply connected spaces $Y$ such that $\Omega Y$ is homotopy equivalent to $S^{3}\left\{2^{r}\right\}$, the homotopy fibre of a degree $2^{r}$ map on the sphere $S^{3}$. In particular showing that such actions do not exist would imply that the homotopy type of $B Q_{2^{n}}$ is determined by its cohomology for all $n$.

Proposition 1.4. Let $G=S D_{2^{n}}$ denote the semidihedral group of order $2^{n}$. Let $X$ be a nilpotent space, comparable to $B G$. Then $\pi_{1} X \cong S D_{2^{k}}$ for some $k \geq 4$.

In section 2 below we give a precise definition of what it means for a space $X$ to be determined by its cohomology. If one considers cohomology as a functor from the homotopy category to the category $\mathcal{K}_{\beta}$, then the class of spaces which are determined by cohomology is understood to be the subclass of the homotopy category, on which the restriction of this functor is 1-1 when considered only as an 
operation on objects. Thus one might wonder whether this class is closed under simple operations such as products, wedges etc. It is hard to believe that one could produce successful answers to those questions in general. However in our more rigid context we are able to prove the following.

Proposition 1.5. Let $G$ be a finite p-group such that the homotopy type of $B G$ is determined by cohomology. Then for any elementary abelian p-group E, the same holds for $B(G \times E)$.

Next we restrict our attention to the isomorphism type of the groups under consideration.

Definition 1.6. Let $\mathcal{D}_{p}$ denote the class of finite $p$-groups which are determined among finite $p$-groups by their mod-p cohomology as objects of $\mathcal{K}_{\beta}$. Namely if $G \in$ $\mathcal{D}_{p}$ and $\pi$ is another finite p-group, then comparability of BG and B $\pi$ implies that $G$ is isomorphic to $\pi$.

With this definition we are now able to state a corollary of our results.

Corollary 1.7. Finite abelian 2-groups, extra special 2-groups, dihedral quaternion and semidihedral groups belong to the class $\mathcal{D}_{2}$. Furthermore, for any prime $p$, the class $\mathcal{D}_{p}$ is closed under direct products with finite elementary abelian p-groups.

It is not hard to observe that in fact, the isomorphism type of $G$, for $G$ an extra special $p$-group, $p$ odd, is determined by the cohomology algebra of $B G$. This result can be extended to any $p$-group of nilpotency class 2 under certain favorable hypotheses.

The main task of this paper is the observation that cohomology as an object of the category $\mathcal{K}_{\beta}$ can sometimes determine the homotopy type of $B G$ for finite nonabelian 2-groups $G$ and indeed, where considering it as an object in the categories $\mathcal{K}$ or $\mathcal{U}$ of unstable algebras or modules over the Steenrod algebra fails to do so.

The authors are grateful to the Centre de Recerca Matemàtica, Barcelona, and the Mathematisches Institut der Universität Heidelberg for their kind hospitality and support while part of this research was done. We also thank Jaume Aguadé, Carles Casacuberta, Richard Kane, Ian Leary, Björn Schuster and Saïd Zarati for useful conversations.

\section{Conventions And Terminology}

All spaces considered are assumed to have the homotopy type of a CW complex and to be 2-complete in the sense of Bousfield and Kan [6]. We denote $H^{*}\left(-; \mathbb{F}_{2}\right)$ by $H^{*}(-)$. Let $\mathcal{A}$ denote the mod-2 Steenrod algebra. Let $\mathcal{K}$ denote the category of unstable algebras over $\mathcal{A}$. By "EMSS" we abbreviate the Eilenberg-Moore spectral sequence and by "SSS" the Serre spectral sequence, both for mod-2 cohomology, unless other coefficients are specified. The Bockstein spectral sequence for mod-2 cohomology is abbreviated by "BSS". The following definitions are included here in order to provide a systematic background for our discussion. The full generality of the concepts we define will not be used in this note but they may become useful in a more general study of the problems suggested here.

Definition 2.1. Let $K$ be an unstable algebra over $\mathcal{A}$. A BSS for $K$ is a spectral sequence $\left\{E_{i}(K), \beta_{i}\right\}_{i=1}^{\infty}$ of differential graded algebras where the differentials have degree one and such that (see $[7,11])$ 
(1) $E_{1}(K)=K$ and $\beta_{1}=S q^{1}$ is the primary Bockstein operator.

(2) If $x \in K^{\text {even }}$ and $x^{2} \neq 0$ in $E_{2}(K)$, then $\beta_{2}\left(x^{2}\right)=x S q^{1} x+S q^{|x|} S q^{1} x$.

(3) If $x \in E_{i}(K)^{\text {even }}$ and $x^{2} \neq 0$ in $E_{i+1}(K), i \geq 2$, then $\beta_{i+1}\left(x^{2}\right)=x \beta_{i}(x)$.

Let $\mathcal{K}_{\beta}$ denote the category whose objects are pairs $\left(K ;\left\{E_{i}(K), \beta_{i}\right\}_{i=1}^{\infty}\right)$, where $K$ is an unstable $\mathcal{A}$-algebra and $\left\{E_{i}(K), \beta_{i}\right\}_{i=1}^{\infty}$ is a BSS for $K$. We will frequently abbreviate an object $\left(K ;\left\{E_{i}(K), \beta_{i}\right\}_{i=1}^{\infty}\right)$ of $\mathcal{K}_{\beta}$ by $K_{\beta}$. A morphism $f: K_{\beta} \longrightarrow K_{\beta}^{\prime}$ in $\mathcal{K}_{\beta}$ is a family of morphisms $\left\{f_{i}\right\}_{i=1}^{\infty}$, where $f_{1}: K \longrightarrow K^{\prime}$ is a morphism of $\mathcal{A}$ algebras and for each $i \geq 2, f_{i}: E_{i}(K) \longrightarrow E_{i}\left(K^{\prime}\right)$ is a morphism of differential graded algebras, which as a morphism of graded algebras, is induced by $f_{i-1}$. The mod-2 cohomology of a space $X$ together with its BSS is a typical object of the category $\mathcal{K}_{\beta}$ that will be denoted by $H_{\beta}^{*}(X)$. Thus mod-2 cohomology can be thought of as a functor from the category of spaces to the category $\mathcal{K}_{\beta}$.

This category has been considered in [2] and [3]. Examples of spaces $X$ with $H^{*}(X) \cong P\left[x_{2}\right] \otimes E\left[y_{3}\right]$, realizing every BSS, which could possibly be associated to this unstable algebra by our set of axioms, are discussed in [2]. Thus we regard (1), (2) and (3) above as a reasonable set of axioms for a BSS associated to an unstable algebra over the Steenrod algebra.

Definition 2.2. Two spaces $X$ and $Y$ are said to be comparable if $H_{\beta}^{*}(X)$ and $H_{\beta}^{*}(Y)$ are isomorphic objects of the category $\mathcal{K}_{\beta}$. We say that $X$ is determined by cohomology if given a space $Y$ comparable to $X$, there is a homotopy equivalence $X \simeq Y$.

Definition 2.3. Let $K_{\beta}$ be an object in $\mathcal{K}_{\beta}$. Let $K$ be the underlying unstable algebra. We say that $K_{\beta}$ is weakly generated by $x_{1}, \cdots, x_{n} \in K$ if any endomorphism $f$ of $K_{\beta}$, such that the restriction of $f_{1}$ to the vector subspace of $K$ spanned by $x_{1}, \cdots, x_{n}$ is an isomorphism, is an isomorphism in $\mathcal{K}_{\beta}$. In that case we shall say that $x_{1}, \cdots, x_{n}$ is a set of generators for $K_{\beta}$ in $\mathcal{K}_{\beta}$.

Throughout this note we shall be calculating the EMSS of certain fibrations such that the base space has a finite 2 -group as a fundamental group. Thus we shall use the fact that the action of a finite 2 -group on a finite dimensional $\mathbb{F}_{2}$-vector space is always nilpotent and so the EMSS is granted to converge to the right target by [8].

Next we point out that in order to grant that a mod-2 cohomology isomorphism through dimension 2 induces an isomorphism on fundamental groups, one might be tempted to assume that all spaces considered are nilpotent. This assumption is not necessary in most cases. Indeed [6, I.6.2] gives a slightly more restricted result, still suitable for our purposes, without the nilpotency assumption. We will have to assume nilpotency however in section 5 below where this lemma of Bousfield and Kan cannot be applied. A final remark is that in our examples the 2-completeness assumption of the spaces $X$ under consideration is not essential to the construction of maps from $X$ to suitable classifying spaces $B G$. One can define the concept of comparability dropping the 2-completeness assumption and then, assuming that $X$ is comparable to $B G$ for some finite 2 -group $G$, one might be able to construct a map from $X$ to $B G$ and then examine its cohomological properties. If the map happens to induce an isomorphism on cohomology then our assertions would hold for the 2-completion of $X$.

There are odd primary analogues of all concepts we have defined in this section. Only Definition 2.1 needs the obvious reformulation at odd primes. 
Definition 2.4. Let $p$ be an odd prime and $\mathcal{A}_{p}$ the mod-p Steenrod algebra. Let $K$ be an unstable algebra over $\mathcal{A}_{p}$. A BSS for $K$ is a spectral sequence $\left\{E_{i}(K), \beta_{i}\right\}_{i=1}^{\infty}$ of differential graded algebras where the differentials have degree one and such that (see $[7,11])$

(1) $E_{1}(K)=K$ and $\beta_{1}=\beta$ is the primary Bockstein operator.

(2) If $x \in E_{i}(K)^{\text {even }}$ and $x^{p} \neq 0$ in $E_{i+1}(K), i \geq 1$, then $\beta_{i+1}\left(x^{p}\right)=x^{p-1} \beta_{i}(x)$.

\section{The Dihedral Groups}

This section is devoted to the proof of Theorem 1.1. The dihedral group of order 8 is a particular case of an extra special 2-group. However, the calculation here applies to dihedral 2-groups of arbitrary order and is different in nature from the one performed in the proof of Theorem 1.2 below.

Let $D_{2^{n}}$ denote a dihedral group of order $2^{n}$.

$$
D_{2^{n}}=\left\langle x, y \mid x^{2^{n-1}}=y^{2}=1 ; y x y=x^{-1}\right\rangle .
$$

Recall from [10] for $n \geq 3$

$$
H^{*}\left(B D_{2^{n}}\right) \cong P\left[x_{1}, y_{1}, w_{2}\right] /\left(x^{2}+x y\right),
$$

where the degrees are given by the subscripts. The action of the Steenrod algebra is given by $S q^{1}(w)=w y$ and all the other operations are determined by the regular axioms. The Bockstein spectral sequence is determined by the requirement that

$$
E_{2} \cong E_{3} \cong \cdots \cong E_{n-1} \cong P\left[w^{2}\right] \otimes E[x w]
$$

and $\beta_{n-1}(x w)=w^{2}$. Indeed $D_{2^{n}}$ has a normal cyclic subgroup of order $2^{n-1}$, which detects this higher Bockstein. This calculation is completely routine using the SSS for the respective group extension.

Notice that the algebra generators for $H^{*}\left(B D_{2^{n}}\right)$ are the same as the generators in $\mathcal{K}_{\beta}$. Thus for the proof of Theorem 1.1, it suffices to show that any space $X$ comparable to $B D_{2^{n}}$ admits a map $X \longrightarrow B D_{2^{n}}$, inducing an isomorphism on cohomology up to dimension 2 .

Let $X$ be a space comparable to $B D_{2^{n}}$. Let $D_{4}$ denote an elementary abelian 2-group of rank 2. There is a map $\phi_{2}: X \longrightarrow B D_{4}$, classifying the classes $x, y \in$ $H^{1}(X)$. Consider the tower of principal fibrations

$$
\cdots \longrightarrow B D_{2^{n}} \stackrel{\pi_{n-1}}{\longrightarrow} B D_{2^{n-1}} \stackrel{\pi_{n-2}}{\longrightarrow} \cdots \stackrel{\pi_{3}}{\longrightarrow} B D_{8} \stackrel{\pi_{2}}{\longrightarrow} B D_{4} .
$$

Each projection $\pi_{i}$ corresponds to the central extension

$$
0 \longrightarrow \mathbb{Z} / 2 \longrightarrow D_{2^{i+1}} \longrightarrow D_{2^{i}} \longrightarrow 1
$$

classified by $w \in H^{2}\left(B D_{2^{i}}\right)$ if $i \geq 3$ and by $x^{2}+x y \in H^{2}\left(B D_{4}\right)$ if $i=2$.

Lemma 3.1. The map $\phi_{2}: X \longrightarrow B D_{4}$ factors through $B D_{2^{n}}$.

Proof. Clearly the composite

$$
X \stackrel{\phi_{2}}{\longrightarrow} B D_{4} \stackrel{x^{2}+x y}{\longrightarrow} K(\mathbb{Z} / 2,2)
$$

is null-homotopic. Hence $\phi_{2}$ factors through $B D_{8}$. Thus we must show that the composite

$$
X \stackrel{\phi_{n-k}}{\longrightarrow} B D_{2^{n-k}} \stackrel{w}{\longrightarrow} K(\mathbb{Z} / 2,2)
$$

is null-homotopic for $k \geq 1$. 
Indeed it is easy to see by applying naturality of $S q^{1}$ that $\phi_{n-k}^{*}(w)=a w$ for some $a \in \mathbb{F}_{2}$. If $a=1$ then $\phi_{n-k}^{*}$ is an isomorphism, contradicting the existence of different Bockstein operators in $H^{*}(X)$ and $H^{*}\left(B D_{2^{n-k}}\right)$. Thus $\phi_{n-k}^{*}(w)=0$ as required.

To complete the proof of Theorem 1.1 we must show that $\phi_{n}^{*} w=w$. Suppose $\phi_{n}^{*} w=0$. An easy EMSS calculation for the fibration

$$
F \stackrel{j}{\longrightarrow} X \stackrel{\phi_{n}}{\longrightarrow} B D_{2^{n}}
$$

shows that

$$
H^{*}(F) \cong P\left[v_{2}\right] \otimes E\left[\alpha_{1}\right]
$$

as a $P[v]$-module, where $v=j^{*}(w) \in H^{2}(X)$.

Since $\beta_{n-1}(x w)=w^{2}$ in $H_{\beta}^{*}(X)$ and $j^{*}(x)=0$, it follows by naturality of the $B S S$ that $\beta_{r}(\alpha v)=v^{2}$ in $H_{\beta}^{*}(F)$ for some $r<n-1$, which in turn easily implies that $\beta_{r}(\alpha)=v$.

This determines the homotopy type of $F$ to be that of $B \mathbb{Z} / 2^{r}$. Hence $X \simeq B H$, where $H$ fits in a group extension of the form

$$
0 \longrightarrow \mathbb{Z} / 2^{r} \longrightarrow H \longrightarrow B D_{2^{n}} \longrightarrow 1
$$

We argue that $H \cong D_{2^{n+r}}$ and having done so, we obtain a contradiction to our hypothesis, for if $X \cong B D_{2^{n+r}}, r \geq 1$ then $H^{*}(X)$ gives rise to a different object of $\mathcal{K}_{\beta}$ than the one assumed.

Indeed, consider the fibration

$$
B \mathbb{Z} / 2^{r} \longrightarrow X \stackrel{\phi_{n}}{\longrightarrow} B D_{2^{n}} .
$$

Since $\phi_{n}^{*} w=0$, it follows that $\phi_{n}$ lifts to a map $\phi_{n+1}: X \longrightarrow B D_{2^{n+1}}$. One observes immediately that the homotopy fibre of $\phi_{n+1}$ is $B \mathbb{Z} / 2^{r-1}$. The same argument as above implies that $\phi_{n+1}^{*} w=a w$ for some $a \in \mathbb{F}_{2}$. But if $a=1$ then $\phi_{n+1}$ is an equivalence, which would imply $r=1$ and we get the desired contradiction. Thus if $r>1$, then $a=0$ and one proceeds inductively. Notice that the process must stop for $\phi_{n+r}: X \longrightarrow B D_{2^{n+r}}$ has to be an equivalence. This completes the proof of Theorem 1.1.

\section{The Extra Special 2-Groups}

An extra special 2-group is a central extension of an elementary abelian 2-group by a cyclic group of order 2. The cohomology of extra special 2-groups was computed by Quillen [14]. We start by recalling Quillen's result. A good general reference for the subject is [4].

Let $G$ be an extra special 2-group. Thus $G$ fits in a central extension

$$
0 \longrightarrow \mathbb{Z} / 2 \longrightarrow G \longrightarrow E \longrightarrow 0
$$

where $E$ is an elementary abelian 2-group of rank $n$. Write

$$
H^{*}(B E) \cong P\left[x_{1}, x_{2}, \cdots, x_{n}\right] .
$$

Then the extension (4.1) is classified by some quadratic form $q \in H^{2}(B E)$.

Theorem 4.1 (Quillen). Let $G$ be as above. Then

$$
H^{*}(B G) \cong P\left[x_{1}, x_{2}, \cdots, x_{n}\right] /\left(q_{0}, q_{1}, \cdots, q_{h}\right) \otimes P[\zeta]
$$


with $q_{0}=q, q_{1}=S q^{1} q_{0}$ and $q_{i}=S q^{2^{i-1}} q_{i-1}$. The elements $x_{1}, x_{2}, \cdots, x_{n}$ are degree 1 elements inflated from $H^{1}(B E)$. The number $2^{h}$ can be interpreted as the index of a maximal elementary abelian subgroup of $G$. The element $\zeta$ has degree $2^{h}$ and can be chosen to be any element of this degree, restricting non-trivially to the kernel in the extension (4.1) above.

The following theorem deals with the Bockstein spectral sequence for $H^{*}(B G)$ and is proven in [4].

Theorem 4.2. Let $G$ be an extra special 2-group with $h \geq 2$. Then the $E_{2}$ page of the Bockstein spectral sequence for $H^{*}(B G)$ is given by

$$
\begin{gathered}
E_{2}^{*}=E\left[\gamma_{1}, \gamma_{3}, \gamma_{4} \cdots, \gamma_{h}\right] \otimes P[\zeta] \\
\text { with }\left|\gamma_{1}\right|=3,\left|\gamma_{j}\right|=2^{j-1} \text { for } j \geq 3 \text { and }|\zeta|=2^{h} . \\
\text { For } 3 \leq r \leq h \text { we have, } \\
\qquad E_{r}^{*}=E\left[\gamma_{1} \gamma_{3} \cdots \gamma_{r}, \gamma_{r+1}, \cdots, \gamma_{h}\right] \otimes P[\zeta] .
\end{gathered}
$$

Finally, $E_{h+1}^{*}=E_{h}^{*}$ and $E_{r}^{*}=\mathbb{F}_{2}$ for $r \geq h+2$.

The differentials are given by $\beta_{2}\left(\gamma_{1}\right)=\gamma_{3}, \beta_{r}\left(\gamma_{1} \gamma_{3} \cdots \gamma_{r}\right)=\gamma_{r+1}, \beta_{r}\left(\gamma_{i}\right)=0$ for $r<i, \beta_{r}(\zeta)=0$ for all $r$ and $\beta_{h+1}\left(\gamma_{1} \gamma_{3} \cdots \gamma_{h}\right)=\zeta$.

Notice that the case $h=0$ corresponds to elementary abelian 2-groups and $h=1$ to $\mathbb{Z} / 4 \times E$ or $D_{8} \times E$ where $E$ is elementary abelian. The first case is abelian and thus obvious. We will remark about $D_{8} \times E$ later.

Lemma 4.3. Let $G$ be an extra special 2-group with $h \geq 2$. Then $H_{\beta}^{*}(B G)$ is weakly generated by $H^{1}(B G)$.

Proof. Let $K_{\beta}$ denote $H_{\beta}^{*}(B G)$. Let $f$ be an endomorphism of $K_{\beta}$, such that the corresponding endomorphism $f_{1}$ of $K$, restricted to dimension 1 , is an isomorphism. We must show that $f$ is an isomorphism.

Assume that $G$ is an extra special 2-group, given by an extension on the form (4.1), whose cohomology is given by Theorems 4.1 and 4.2 . Without loss of generality we may assume that

$$
f_{1}\left(x_{i}\right)=x_{i} \text { for all } i
$$

Indeed, $f_{1}$ restricted to dimension 1 being an isomorphism, it is obviously an isomorphism when restricted to $P\left[x_{1}, x_{2}, \cdots, x_{n}\right] /\left(q_{0}, q_{1}, \cdots, q_{h}\right)$. In particular, the unique extension of $f_{1}$ to $P\left[x_{1}, x_{2}, \cdots, x_{n}\right] \cong H^{*}(B E)$ preserves the quadratic form $q$ that classifies the extension (4.1). Regarding $f_{1}$, restricted to dimension 1 , as the dual of an automorphism $\tilde{f}_{1}$ of $E$, one sees that $\tilde{f}_{1}$ extends to an automorphism $\tilde{f}_{1}: G \rightarrow G$. The induced map on cohomology $\tilde{f}_{1}^{*}$ is an isomorphism with the property that $\tilde{f}_{1}^{*}\left(x_{i}\right)=f_{1}\left(x_{i}\right)$. Hence the map $f^{\prime}:=\left(\tilde{f}_{1}^{-1}\right)^{*} \circ f$ is an endomorphism of $H_{\beta}^{*}(B G)$, satisfying condition (4.2) above.

So, assuming (4.2), it follows easily that $f_{r}$ maps the exterior generators in $E_{r}^{*}(K)$ to themselves for all $r$. Thus we get at the $h+1$ page of the spectral sequence

$$
f_{h+1}(\zeta)=f_{h+1} \beta_{h+1}\left(\gamma_{1} \gamma_{3} \cdots \gamma_{h}\right)=\beta_{h+1} f_{h+1}\left(\gamma_{1} \gamma_{3} \cdots \gamma_{h}\right)=\zeta
$$

On the other hand $f_{j}$ is induced by $f_{j-1}$. Thus we must have $f_{1}(\zeta)=\zeta+p$, for some polynomial $p=p\left(x_{1}, \cdots, x_{n}\right)$. By Theorem 4.1 the self map of $K$ which takes $x_{i}$ to itself for every $i$ and $\zeta$ to $\zeta+p$ is an isomorphism, hence the proof is complete. 
We are now ready to prove Theorem 1.2. Assume that $X$ is a space with

$$
H^{*}(X) \cong H^{*}(B G)
$$

as algebras, where $G$ is an extra special 2 -group of order $2^{n+1}$ classified by the quadratic form $q$ and with maximal elementary abelian 2 -subgroup of index $2^{h}$. We will show:

(1) If $h \geq 3$, then $X \simeq B G$.

(2) If $h \geq 1$ and $H_{\beta}^{*}(X) \cong H_{\beta}^{*}(B G)$, then $X \simeq B G$.

We start by proving (1). Suppose $h \geq 3$ and let $X$ be a space such that $H^{*}(X) \cong$ $H^{*}(B G)$ as algebras. Let $G$ be given by the central extension (4.1). The argument above yields that any map $X \stackrel{p}{\longrightarrow} B E$ inducing an isomorphism on $H^{1}(-)$, lifts to a map $X \stackrel{\psi}{\longrightarrow} B G$ such that $\psi^{*}$ is an isomorphism up to dimension $2^{h}-1$. Furthermore, it is possible to choose $\psi$ such that under a suitable change of basis, $\psi^{*}(\zeta)=\epsilon \zeta$ for some $\epsilon \in \mathbb{F}_{2}$. If $\epsilon=1$, then $\psi^{*}$ is an isomorphism. Thus assume that $\epsilon=0$.

The EMSS for the fibration

$$
F \longrightarrow X \stackrel{\psi}{\longrightarrow} B G
$$

is easily shown to collapse at $E_{2}$ and

$$
E_{2} \cong E_{\infty} \cong P[v] \otimes E[\alpha]
$$

with $|v|=2^{h}$ and $|\alpha|=2^{h}-1$, as a $P[v]$-module. By [1] it follows that either $|v|=2$ or $|v|=4$. Hence we are in the case $h \leq 2$, contradicting our hypothesis.

Next we turn to the proof of (2). Consider the case $h \geq 2$. Indeed, there is a map $X \stackrel{p}{\longrightarrow} B E$ corresponding to the projection $B G \stackrel{\pi}{\longrightarrow} B E$. Since the composite

$$
X \stackrel{p}{\longrightarrow} B E \stackrel{q}{\longrightarrow} K(\mathbb{Z} / 2,2)
$$

is null-homotopic, there is a map $X \stackrel{\psi}{\longrightarrow} B G$, lifting $p$. Moreover, since $p$ induces an isomorphism in 1-dimensional cohomology and $h \geq 2$, it follows that any such lift induces an isomorphism in cohomology up to dimension $2^{h}-1 \geq 3$. The result follows by Lemma 4.3.

For $h=1$ it remains to consider the case, where $G \cong D_{8} \times E$ with $E$ elementary abelian. This case is a consequence of Theorem 1.1 proved in the previous section and Proposition 1.5 which is proved in section 7.

This completes the proof of Theorem 1.2.

Remark 4.4. Notice that the conclusion $X \simeq B G$ is not true for $h=1$ unless $H_{\beta}^{*}(X) \cong H_{\beta}^{*}(B G)$, for dihedral groups of different orders produce counterexamples. We are not aware of a suitable example for $h=2$.

\section{The Quaternion Groups $Q_{2^{n}}$}

The quaternion groups are given as follows:

$$
Q_{2^{n}}=\left\langle x, y \mid x^{2^{n-2}}=y^{2} ; x^{2^{n-1}}=1 ; y x y^{-1}=x^{-1}\right\rangle .
$$

Recall the cohomological structure of $B Q_{2^{n}}$ :

$$
H^{*}\left(B Q_{2^{n}}\right) \cong P\left[x_{1}, y_{1}, v_{4}\right] /(\alpha, \beta)
$$

where $\alpha=x^{2}+x y+y^{2}, \beta=x^{2} y+y^{2} x$ if $n=3$ and $\alpha=x^{2}+x y, \beta=y^{3}$ if $n \geq 4$. The Bockstein spectral sequence is determined by the regular axioms for $S q^{1}$ and the 
requirement that $\beta_{n}(c)=v$, where $c \in H^{3}\left(B Q_{2^{n}}\right)$ is the unique generator. Notice that the $n$-th Bockstein is defined here with no indeterminacy and that the class $c$ is a product of 1 -dimensional generators. Thus $H^{*}\left(B Q_{2^{n}}\right)$ is weakly generated by $x$ and $y$ as an object of $\mathcal{K}_{\beta}$.

The central extension

$$
0 \longrightarrow \mathbb{Z} / 2 \longrightarrow Q_{2^{n}} \longrightarrow D_{2^{n-1}} \longrightarrow 0
$$

is characterized by the class $q_{2} \in H^{*}\left(B D_{2^{n-1}}\right)$, where $q_{2}=x^{2}+x y+y^{2}$ if $n=3$ and $q_{2}=w+y^{2}$ if $n \geq 4$ (see for example [9]).

The group $Q_{8}$ is an extra special 2-group. Thus we will assume $n \geq 4$.

Proposition 5.1. Suppose $X$ is comparable to $B Q_{2^{n}}$ for some $n \geq 4$. Then $\pi_{1} X$ is isomorphic to $Q_{2^{k}}$ for some $4 \leq k \leq n$. In particular if $n=k$, then $X \simeq B Q_{2^{n}}$.

Proof. There is a map $\phi_{2}: X \longrightarrow B D_{4}$, where $D_{4}=\mathbb{Z} / 2 \oplus \mathbb{Z} / 2$, classifying $x, y \in H^{*}(X)$. Consider the tower of principal fibrations

$$
\cdots \longrightarrow B D_{2^{n}} \stackrel{\pi_{n-1}}{\longrightarrow} B D_{2^{n-1}} \stackrel{\pi_{n-2}}{\longrightarrow} \cdots \stackrel{\pi_{3}}{\longrightarrow} B D_{8} \stackrel{\pi_{2}}{\longrightarrow} B D_{4} .
$$

Recall that the map $\pi_{2}$ is classified by the class $x^{2}+x y \in H^{2}\left(B D_{4}\right)$ and that each map $\pi_{i}$ for $i \geq 3$ is classified by $w \in H^{2}\left(B D_{2^{i}}\right)$.

Since $\phi_{2}^{*}\left(x^{2}+x y\right)=0, \phi_{2}$ lifts to $\phi_{3}: X \longrightarrow B D_{8}$ inducing isomorphism in 1-dimensional cohomology. Suppose that for some $i \geq 3$ there is a map $\phi_{i}: X \longrightarrow$ $B D_{2^{i}}$ factoring $\phi_{2}$. Then $\phi_{i}^{*}$ is an isomorphism in dimension 1 and by applying naturality of $S q^{1}$ one easily observes that $\phi_{i}^{*}(w)=b y^{2}$ for some $b \in \mathbb{F}_{2}$. If $b=0$, then $\phi_{i}$ lifts further to a map $\phi_{i+1}: X \longrightarrow B D_{2^{i+1}}$ and the argument may be iterated. On the other hand if for some $k \geq 4, \phi_{k-1}^{*}(w)=y^{2}$, then $\phi_{k-1}^{*}\left(w+y^{2}\right)=0$, and the composite

$$
X \stackrel{\phi_{k-1}}{\longrightarrow} B D_{2^{k-1}} \stackrel{w+y^{2}}{\longrightarrow} K(\mathbb{Z} / 2,2)
$$

is null-homotopic. The class $w+y^{2}$ is known to be the extension class for the quaternion group $Q_{2^{n}}$ (see for instance [9]). Thus $\phi_{k-1}$ lifts to a map $\lambda_{k}: X \rightarrow$ $B Q_{2^{k}}$, inducing an isomorphism on cohomology in dimension 1 through 3 . Hence in this case $\pi_{1}(X) \cong Q_{2^{k}}$ for some $k \geq 4$ ([6, I.6.2].) It remains to show that the lifting process actually finishes at some $k \leq n$. Thus suppose that it doesn't. Then $\phi_{i}$ is defined for every $i \geq 2$ and $\phi_{i}^{*}(w)=0$. Define $F_{i}$ to be the homotopy fibre of $\phi_{i}$, thus obtaining a sequence of fibrations

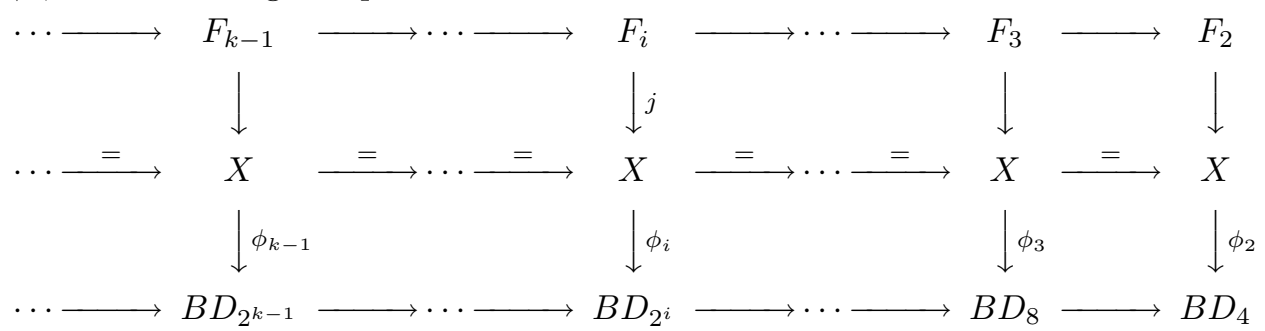

An EMSS calculation yields

$$
H^{*} F_{i} \cong E\left[a_{1}, a_{2}\right] \otimes P\left[v_{4}\right]
$$

as $P\left[v_{4}\right]$-modules, where $v_{4}$ is the restriction of $v_{4} \in H^{4}(X)$. Moreover by naturality of the EMSS, the map $F_{i+1} \longrightarrow F_{i}$ induces an isomorphism in even dimensional cohomology and it is trivial in odd dimensions. 
Now consider each fibration separately. Since $\beta_{n}\left(x^{3}\right)=v$ in $H_{\beta}^{*}(X)$, we obtain that $\beta_{r_{i}}\left(a_{1} a_{2}\right)=v$ in $H^{*}\left(F_{i}\right)$ for some $r_{i}<n$ and therefore $\beta_{s_{i}}\left(a_{1}\right)=a_{2}$ for some $s_{i} \leq r_{i}$. If $s_{i}=r_{i}$ for some $i$, then $F_{i}$ is comparable to $B \mathbb{Z} / 2^{r_{i}}$ and thus $F \simeq B \mathbb{Z} / 2^{r_{i}}$. In that case a similar argument to the one presented in the proof of Theorem 1.1 shows that $X \simeq B Q_{2^{i+r_{i}}}$, which in turn implies that $i+r_{i}=n$ by considering the action of the higher Bockstein operators, distinguishing between classifying spaces of different quaternion groups. Assume now $s_{i}<r_{i}$ for all $i$. Comparing cohomology of $F_{i+1}$ and $F_{i}$, we observe that $r_{i+1}<r_{i}$. Hence, at the $(k-1)$-st step we have a sequence of inequalities:

$$
1 \leq s_{k-1}<r_{k-1}<\cdots<r_{3}<r_{2}<n
$$

from which it follows that the lifting process has to stop at some $k, 4 \leq k \leq n$.

Corollary 5.2. The homotopy type of $B Q_{16}$ is determined by cohomology.

Proposition 5.3. Let $X$ be a space comparable to $B Q_{2^{n}}$ with $\pi_{1} X \cong Q_{2^{k}}$ for some $4<k<n$, and let $\tilde{X}$ be its universal cover, then

(a) $H_{\beta}^{*}(\tilde{X})=E\left[z_{3}\right] \otimes P\left[v_{4}\right]$ with $\beta_{n-k}\left(z_{3}\right)=v_{4}$.

(b) The action of $Q_{2^{k}}$ on $H^{*}(\tilde{X}, \mathbb{Z})$ is trivial.

(c) The quotient space $\tilde{X} / Q_{2^{k-1}}$ is comparable to $B Q_{2^{n-1}}$.

Proof. The EMSS for $\tilde{X} \longrightarrow X \longrightarrow B Q_{2^{k}}$ shows that

$$
H^{*} \tilde{X}=E\left[z_{3}\right] \otimes P\left[v_{4}\right]
$$

where $v_{4}$ is the restriction of $v_{4} \in H^{4}(X)$. Furthermore, by naturality of the BSS, $\beta_{t}\left(z_{3}\right)=v_{4}$ for some $t<n$. It follows that $\tilde{X}$ is of finite type [2, Proposition 5.7] and that the rings $H^{*}(\tilde{X} ; \mathbb{Z})$ and $\mathbb{Z} / 2^{t}\left[\bar{v}_{4}\right]$ have isomorphic augmentation ideals. The generator $\bar{v}_{4}$ reduces to $v_{4}$ mod 2. We proceed in proving (c). The proofs of (a) and (b) will follow.

There is a homomorphism $Q_{2^{n}} \longrightarrow \mathbb{Z} / 2$ given by sending $x$ to the generator of $\mathbb{Z} / 2$ and $y$ to 1 . The kernel of this homomorphism is a quaternion subgroup of $Q_{2^{n}}$ of order $2^{n-1}$. Let $X \stackrel{\alpha}{\longrightarrow} B Q_{2^{k}}$ denote the map classifying the universal cover for $X$. Then there is a commutative diagram

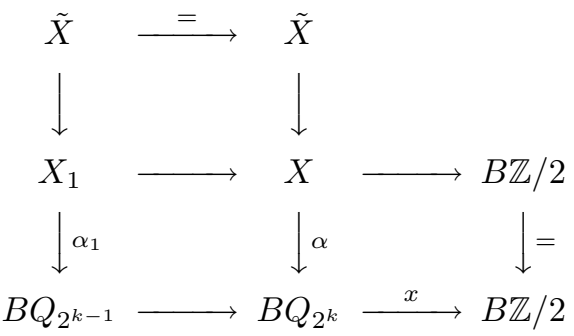

Calculating the EMSS for (A) and (B) and using naturality, it follows that $H^{*}\left(X_{1}\right)$ $\cong H^{*}\left(B Q_{2^{n-1}}\right)$ as an algebra. Obviously $\pi_{1} X_{1} \cong Q_{2^{k-1}}$. Moreover by naturality of the BSS, $\beta_{r}\left(x^{3}\right)=v_{4}$ in $H^{*}\left(X_{1}\right)$, for some $r \leq n-1$. Thus $X_{1}$ is comparable to $B Q_{2^{r}}$.

Next consider the integral SSS for (A).

$$
E_{2}^{p, q} \cong H^{p}\left(\mathbb{Z} / 2 ; H^{q}\left(X_{1} ; \mathbb{Z}\right)\right) \text {. }
$$

In particular $E_{2}^{p, 2} \cong H^{p}(\mathbb{Z} / 2 ; \mathbb{Z} / 2 \oplus \mathbb{Z} / 2)$ and by inspection of the spectral sequence it follows that the action of $\mathbb{Z} / 2$ is the twisting action. Thus $E_{2}^{0,2} \cong \mathbb{Z} / 2$ and 
$E_{2}^{p, 2}=0$ if $p \geq 1$. Consequently $E_{2}^{0,4} \cong\left(H^{4}\left(X_{1} ; \mathbb{Z}\right)\right)^{\mathbb{Z} / 2} \cong \mathbb{Z} / 2^{n-1}$ and since $H^{4}\left(X_{1}, \mathbb{Z}\right) \cong \mathbb{Z} / 2^{r}, r \leq n-1$, we get both that $r=n-1$ and that the action is trivial. This completes the proof of part (c).

Consider the commutative diagram

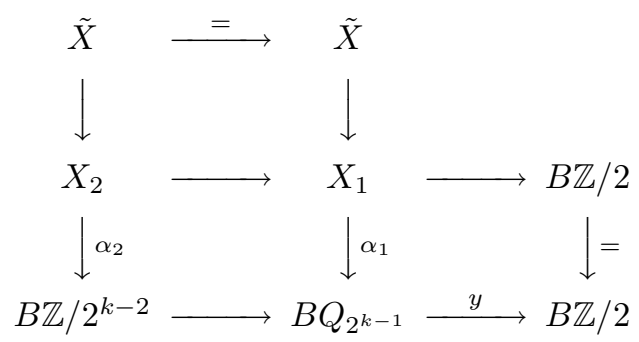

Again the EMSS is employed to compute $H^{*}\left(X_{2}\right)$. By naturality we get

$$
H^{*}\left(X_{2}\right) \cong P\left[v_{4}\right] \otimes E\left[x_{1}\right] \otimes P\left[u_{2}\right] /\left(u^{2}\right)
$$

where $v_{4}$ is restriction of the corresponding class in $H^{*}\left(X_{1}\right)$ and $x_{1}$ and $u_{2}$ are inflated from $H^{*}\left(B \mathbb{Z} / 2^{k-1}\right)$ via $\alpha_{2}^{*}$. The structure given above is easily seen to be both the $P\left[v_{4}\right]$-module structure and the $H^{*}\left(B \mathbb{Z} / 2^{k-1}\right) \cong E\left[x_{1}\right] \otimes P\left[u_{2}\right]$-module structure. Using naturality of the BSS, it follows that $\beta_{k-1}(x)=u$ and $\beta_{s}(x u)=v$, for some $s<n-1$. We proceed in computing the Bockstein spectral sequence for $H^{*}\left(X_{2}\right)$. Consider the SSS with integral coefficients for the fibration (C) above.

$$
E_{2}^{p, q} \cong H^{p}\left(B \mathbb{Z} / 2 ; H^{q}\left(X_{2} ; \mathbb{Z}\right) \Rightarrow H^{p+q}\left(X_{1} ; \mathbb{Z}\right) .\right.
$$

In total degree two we have

$$
\begin{gathered}
E_{2}^{2,0}=H^{2}\left(B \mathbb{Z} / 2 ; H^{0}\left(X_{2} ; \mathbb{Z}\right)\right) \cong \mathbb{Z} / 2, \\
E_{2}^{0,2}=H^{0}\left(B \mathbb{Z} / 2 ; H^{2}\left(X_{2} ; \mathbb{Z}\right)\right) \cong H^{0}\left(B \mathbb{Z} / 2 ; \mathbb{Z} / 2^{k-1}\right) \cong\left(\mathbb{Z} / 2^{k-1}\right)^{\mathbb{Z} / 2} .
\end{gathered}
$$

Since $H^{2}\left(X_{1} ; \mathbb{Z}\right) \cong \mathbb{Z} / 2 \oplus \mathbb{Z} / 2$, it follows that $\mathbb{Z} / 2$ acts non-trivially on $\mathbb{Z} / 2^{k-1}$ and $E_{2}^{0,2} \cong \mathbb{Z} / 2$.

In total degrees three and four we have:

$$
\begin{gathered}
E_{2}^{1,2}=H^{1}\left(B \mathbb{Z} / 2 ; H^{2}\left(X_{2} ; \mathbb{Z}\right)\right)=H^{1}\left(B \mathbb{Z} / 2 ; \mathbb{Z} / 2^{k-1}\right), \\
E_{2}^{4,0}=H^{4}\left(B \mathbb{Z} / 2 ; H^{0}\left(X_{2} ; \mathbb{Z}\right)\right)=H^{4}(B \mathbb{Z} / 2 ; \mathbb{Z}), \\
E_{2}^{2,2}=H^{2}\left(B \mathbb{Z} / 2 ; H^{2}\left(X_{2} ; \mathbb{Z}\right)\right)=H^{2}\left(B \mathbb{Z} / 2 ; \mathbb{Z} / 2^{k-1}\right), \\
E_{2}^{0,4}=H^{0}\left(B \mathbb{Z} / 2 ; H^{4}\left(X_{2} ; \mathbb{Z}\right)\right)=H^{0}\left(\mathbb{Z} / 2 ; \mathbb{Z} / 2^{r}\right)=\left(\mathbb{Z} / 2^{s}\right)^{\mathbb{Z} / 2} .
\end{gathered}
$$

Since $H^{3}\left(X_{1} ; \mathbb{Z}\right)=0$ it follows that $E_{3}^{1,2} \cong \mathbb{Z} / 2$ and $d_{3}: E_{3}^{1,2} \longrightarrow E_{3}^{4,0}$ is an isomorphism.

Next notice that $E_{3}^{0,4}=\left(\mathbb{Z} / 2^{s}\right)^{\mathbb{Z} / 2}$, where $s<n-1$ and that 2 annihilates the cohomology of $B \mathbb{Z} / 2$ with any coefficients. Since $H^{4}\left(X_{1} ; \mathbb{Z}\right) \cong \mathbb{Z} / 2^{n-1}$, it follows that $E_{3}^{0,4}=\mathbb{Z} / 2^{n-2}$ and $E_{3}^{2,2}=\mathbb{Z} / 2$. Thus $s=n-2$ and the action of $\mathbb{Z} / 2$ on $H^{4}\left(X_{2} ; \mathbb{Z}\right)$ is trivial. This completes the calculation of the BSS for $H^{*}\left(X_{2}\right)$.

Now, we are ready to compute the BSS for $H^{*}(\tilde{X})$. Again we use the SSS with integral coefficients for the maps $\alpha, \alpha_{1}$ and $\alpha_{2}$ in the diagrams above. In total degree four, these spectral sequences give the following extensions:

$$
\begin{array}{ll}
\alpha: & \mathbb{Z} / 2^{k} \rightarrow \mathbb{Z} / 2^{n} \rightarrow\left(\mathbb{Z} / 2^{t}\right)^{Q_{2^{k}}} \\
\alpha_{1}: & \mathbb{Z} / 2^{k-1} \rightarrow \mathbb{Z} / 2^{n-1} \rightarrow\left(\mathbb{Z} / 2^{t}\right)^{Q_{2^{k-1}}} \\
\alpha_{2}: & \mathbb{Z} / 2^{k-2} \rightarrow \mathbb{Z} / 2^{n-2} \rightarrow\left(\mathbb{Z} / 2^{t}\right)^{\mathbb{Z} / 2^{k-2}} .
\end{array}
$$


It follows that

$$
\left(\mathbb{Z} / 2^{t}\right)^{Q_{2^{k}}} \cong\left(\mathbb{Z} / 2^{t}\right)^{Q_{2^{k-1}}} \cong\left(\mathbb{Z} / 2^{t}\right)^{\mathbb{Z} / 2^{k-2}} \cong \mathbb{Z} / 2^{n-k},
$$

where the groups $\mathbb{Z} / 2^{k-2} \subset Q_{2^{k-1}}$ operate on $\mathbb{Z} / 2^{t}$ as subgroups of $Q_{2^{k}}$. But $\operatorname{Aut}\left(\mathbb{Z} / 2^{t}\right)$ is abelian and then the action of $Q_{2^{k}}$ factors through its abelianization: $\left(Q_{2^{k}}\right)_{a b} \cong \mathbb{Z} / 2 \oplus \mathbb{Z} / 2$. Since $\mathbb{Z} / 2^{k-2}$, generated by $x^{2} \in Q_{2^{k}}$, is contained in the kernel of the abelianization, its action is trivial. But the action of $Q_{2^{k}}$ on $\mathbb{Z} / 2^{t}$ has the same invariants as the action of $\mathbb{Z} / 2^{k-2}$. Hence $t=n-k$ and $Q_{2^{k}}$ operates trivially. This completes the proof of (a) and (b).

Corollary 5.4. Let $X$ be comparable to $B Q_{2^{n}}$ with $\pi_{1} X \cong Q_{2^{k}}$ for some $4<k<$ $n$. Then for every $1 \leq j \leq k-4$, there exists a space $X_{j}$ comparable to $B Q_{2^{n-j}}$ with $\pi_{1} X_{j} \cong Q_{2^{k-j}}$.

Corollary 5.5. Let $X$ be comparable to $B Q_{2^{n}}$ for some $n \geq 5$. Then $\pi_{1} X \neq$ $Q_{2^{n-1}}$. Consequently the homotopy type of $B Q_{32}$ is determined by its cohomology.

Proof. By Proposition 5.3 assuming the contrary implies that the universal cover $\tilde{X}$ of $X$ satisfies

$$
H^{*}(\tilde{X}) \cong P\left[t_{4}\right] \otimes E\left[x_{3}\right]
$$

with $S q^{1} x=t$. By an argument due to Aguadé [1], a space with such cohomology does not exist.

Finally we remark that proving that the homotopy type of $B Q_{2^{n}}$ is determined by cohomology for all $n$ amounts to showing the non-existence of a faithful action of $Q_{16}$ on spaces of the form $Y_{r}, r \geq 2$, where $Y_{r}$ is a simply connected 2-local space such that

$$
H^{*}\left(Y_{r}\right) \cong P\left[t_{4}\right] \otimes E\left[x_{3}\right]
$$

with $\beta_{r} x=t$. For $r=2$ it is not clear whether or not a space such as $Y_{2}$ exists. Of course non-existence of $Y_{2}$ would imply that if $X$ is comparable to $B Q_{2^{n}}$, then $\pi_{1} X \neq Q_{2^{n-2}}$ and hence the homotopy type of $B Q_{64}$ could be determined by its cohomology. For $r \geq 3$ spaces of the form $Y_{r}$ arise from the 2-completion of $B S L_{2}\left(\mathbb{F}_{q}\right)$ for $q$ odd. However there is no suitable free action of $Q_{16}$ on $B S L_{2}\left(\mathbb{F}_{q}\right)$ because in this case the orbit space would be the classifying space of a finite group and comparable to $B Q_{2^{n}}$ for some $n$. (This implies a contradiction, for groups with periodic mod-2 cohomology are completely classified.) It might still be the case that the 2-completion $B S L_{2}\left(\mathbb{F}_{q}\right)_{2}^{\wedge}$ admits a free $Q_{16}$-action. Nonetheless we find it reasonable to conjecture that the homotopy type of $B Q_{2^{n}}$ is determined by cohomology for all $n$.

\section{The Semidihedral Groups}

The semidihedral group $S D_{2^{n}}$ is given as follows

$$
S D_{2^{n}}=\left\langle x, y \mid x^{2^{n-1}}=y^{2}=1, y x y=x^{2^{n-2}-1}\right\rangle .
$$

Recall from [9]

$$
H^{*}\left(B S D_{2^{n}}\right) \cong P[x, y, u, t] /\left(x^{2}+x y, x u, x^{3}, u^{2}+\left(x^{2}+y^{2}\right) t\right)
$$

where $|x|=|y|=1,|u|=3$ and $|t|=4$. The action of the Steenrod algebra is given by $S q^{1} u=S q^{1} t=0, S q^{2} u=(x+y)(t+y u), S q^{2} t=u^{2}$ and $S q^{2^{i}} u=S q^{2^{i}} t=0$ for $i \geq 2$. The Bockstein spectral sequence is determined by the regular axioms for $S q^{1}$ together with the requirement that $\beta_{n-1} u=t$ in $E_{n-1}$. The calculation of 
$\beta_{n-1} u$ easily follows by observing that the unique subgroup of $S D_{2^{n}}$, isomorphic to $Q_{2^{n-1}}$, detects the classes $u$ and $t$ in $\bmod 2$ cohomology [9]. Thus $H^{*}\left(B S D_{2^{n}}\right)$ is weakly generated by $x, y$ and $u$.

Consider the central extension

$$
0 \longrightarrow \mathbb{Z} / 2 \longrightarrow S D_{2^{n}} \longrightarrow D_{2^{n-1}} \longrightarrow 1
$$

characterized by the class $w+x^{2} \in H^{2}\left(B D_{2^{n-1}}\right)$. Consider the tower of principal fibrations

$$
\cdots \longrightarrow B D_{2^{n}} \stackrel{\pi_{n-1}}{\longrightarrow} B D_{2^{n-1}} \stackrel{\pi_{n-2}}{\longrightarrow} \cdots \stackrel{\pi_{3}}{\longrightarrow} B D_{8} \stackrel{\pi_{2}}{\longrightarrow} B D_{4} .
$$

Define $B D_{\infty}$ as the inverse limit of the tower (6.2). It turns out to be an extension

$$
B \hat{\mathbb{Z}}_{2} \rightarrow B D_{\infty} \rightarrow B D_{4}
$$

and by comparison with any other $B \mathbb{Z} / 2^{n-2} \rightarrow B D_{2^{n}} \rightarrow B D_{4}$ it follows that the only possible differential in the SSS for $(6.3)$ is $d_{2}(u)=x^{2}+x y$, where $u$ is the generator of the mod 2 cohomology of $B \hat{\mathbb{Z}}_{2}$, and therefore

$$
H^{*}\left(B D_{\infty}\right) \cong P[x, y] /\left(x^{2}+x y\right) .
$$

Let $X$ be a space comparable to $B S D_{2^{n}}$. There is an obvious map $\phi_{2}: X \rightarrow B D_{4}$ classifying the classes $x, y \in H^{1}(X)$. Lifting $\phi_{2}$ through the towers (6.2) and (6.1) we will obtain a map $f: X \rightarrow B G$, where $G$ is either semidihedral or $D_{\infty}$, such that the induced map in mod 2 cohomology is an isomorphism in dimensions one and two.

Recall from [5] that a map $f$ is called an $H \mathbb{Z} / 2$-equivalence whenever the induced map on mod 2 cohomology is an isomorphism in dimension 1 and a monomorphism in dimension 2. There exists a localization theory $E^{\mathbb{Z} / 2}$ on the category of groups that invert those maps. Finite 2-groups and inverse limits of finite 2-groups are examples of $E^{\mathbb{Z} / 2}$-local groups.

Proposition 6.1. Let $X$ be a space comparable to $B S D_{2^{n}}$. Then $X$ is $H \mathbb{Z} / 2$ equivalent to either $B S D_{2^{k}}$ for some $k$ or $B D_{\infty}$.

Proof. The map $\pi_{2}$ is classified by $x^{2}+x y \in H^{2}\left(B D_{4}\right)$. But $x^{2}+x y=0$ in $H^{2}(X)$. Hence there is a map $\phi_{3}: X \longrightarrow B D_{8}$ with $\pi_{2} \phi_{3}=\phi_{2}$. Assume that there is a map $\phi_{k}: X \longrightarrow B D_{2^{k}}$ lifting $\phi_{3}$. Clearly $\phi_{k}^{*} x=x, \phi_{k}^{*} y=y$. Using naturality of $S q^{1}$ we find that $\phi_{k}^{*} w=a x^{2}$ for some $a \in \mathbb{F}_{2}$.

If $a=1$ then the composite

$$
X \stackrel{\phi_{k}}{\longrightarrow} B D_{k} \stackrel{w+x^{2}}{\longrightarrow} K(\mathbb{Z} / 2,2)
$$

is null homotopic. Thus $\phi_{k}$ lifts to a map

$$
\psi_{k+1}: X \longrightarrow B S D_{2^{k+1}},
$$

which induces an isomorphism on cohomology up to dimension 2. Thus it is an $H \mathbb{Z} / 2$-equivalence. On the other hand if $a=0$, then $\phi_{k}$ can be lifted further. If $\phi_{k}^{*} w=0$ for all $k$ we get a map $X \longrightarrow B D_{\infty}$ inducing as well isomorphism on cohomology up to dimension 2 , which is therefore an $H \mathbb{Z} / 2$-equivalence.

Lemma 6.2. Let $f: X \rightarrow B G$ be an $H \mathbb{Z} / 2$-equivalence where $G$ and its subgroups are $H \mathbb{Z} / 2$-local. Then the induced map $f_{\#}: \pi_{1} X \rightarrow G$ is an epimorphism. Moreover, if $G$ is a finite 2-group then $\pi_{1} X \cong G$. 
Proof. The induced map $f_{\#}: \pi_{1} X \rightarrow G$ is an $H \mathbb{Z} / 2$-equivalence as well, and therefore $E^{\mathbb{Z} / 2}\left(\pi_{1} X\right) \cong G$. Let $H$ denote the image of $f_{\#}$. Since $H$ is $E^{\mathbb{Z} / 2}$-local by hypothesis, the above isomorphism factors through $H$ and therefore $H=G$; that is, $f_{\#}$ is an epimorphism.

Assume now, that $G$ is a finite 2 -group. In particular the previous hypotheses are satisfied. Let $F$ be the fibre of $f$ and

$$
E_{2}^{*, *} \cong H^{*}\left(B G ; H^{*}\left(F ; \mathbb{F}_{2}\right)\right) \Longrightarrow H^{*}\left(X ; \mathbb{F}_{2}\right)
$$

the SSS for the fibration $F \rightarrow X \rightarrow B G$. Since $f$ induces isomorphism on mod 2 cohomology in dimension one and a monomorphism in dimension two, every element of $E_{2}^{1,0}$ and $E_{2}^{2,0}$ must be a permanent cycle and $E_{2}^{0,1} \cong H^{1}\left(F ; \mathbb{F}_{2}\right)^{G}$ must be trivial. The last being the group of invariants of an $\mathbb{F}_{2}$ vector space by the action of a finite 2-group, it is trivial if and only if $H^{1}\left(F ; \mathbb{F}_{2}\right)$ is itself trivial. But this means that $\pi_{1} F=0$ because $F$ is 2 -complete. It follows that $\pi_{1} X \cong G$.

Corollary 6.3. Let $X$ be a nilpotent space comparable to $B S D_{2^{n}}$. Then $\pi_{1} X$ is isomorphic to $S D_{2^{k}}$ for some $k$. Moreover, the natural map $\psi_{k}: X \longrightarrow B S D_{2^{k}}$ classifying the universal cover for $X$ is either a homotopy equivalence or satisfies $\psi_{k}^{*}(u)=\psi_{k}^{*}(t)=0$.

Proof. In Proposition 6.1 we constructed an $H \mathbb{Z} / 2$-equivalence $f: X \rightarrow B G$, where $G$ is either semidihedral of $D_{\infty}$. It follows from Lemma 6.2 that the induced map $\pi_{1} X \rightarrow G$ is an epimorphism. Then, $G$ cannot be $D_{\infty}$ for in that case $X$ would not be nilpotent. Hence $G$ is semidihedral, which is a finite 2-group, so again by Lemma $6.2 f$ induces $\pi_{1} X \cong G$.

Finally one uses the action of the higher Bockstein operators as well as the action of the Steenrod algebra in order to show that $\psi_{k}$ could only have the cohomological effect described in the proposition, in case it fails to be a homotopy equivalence.

\section{Products with Elementary Abelian Groups}

This section is motivated by the will to understand our subject matter from a different point of view. Namely, we have exhibited examples of finite non-abelian 2-groups $G$ such that the homotopy type of $B G$ is determined by its cohomology. A natural question at that point is whether one can construct new examples of groups $G$ with this property out of old ones. More precisely, given two finite 2-groups (or more generally p-groups) $G$ and $H$ such that the homotopy type of $B G$ and $B H$ is determined by cohomology, can one perform certain group theoretic constructions on $G$ and $H$, thus obtaining a new group $K$, possibly infinite, whose classifying space $B K$ has the same property. One might have in mind constructions such as Cartesian products, amalgamated products, fibre products etc. The simplest possible construction is of course the Cartesian product. Thus one might wonder whether the homotopy type of $B(H \times G)$ is determined by its cohomology if the same holds for each one of the factors.

Proposition 1.5 handles the simplest possible version of this question. We now turn to its proof. Assume that $p$ is any prime. $H_{\beta}^{*}$ is thus assumed to be mod- $p$ cohomology. Let $E$ be an elementary abelian $p$-group and suppose that $X$ is a space comparable to $B(G \times E)$, that is 


$$
H_{\beta}^{*}(X) \cong H_{\beta}^{*}(B(G \times E)) \cong H_{\beta}^{*}(B G) \otimes H_{\beta}^{*}(B E) .
$$

We can obtain a classifying map for one dimensional classes

$$
\phi: X \rightarrow B E
$$

inducing the obvious inclusion $H^{*}(B E) \hookrightarrow H^{*}(B G) \otimes H^{*}(B E)$. Let $F$ be the fibre of $\phi$. Since $H^{*}(X)$ is free as a module over $H^{*}(B E)$, the EMSS for this fibration is concentrated in the zero vertical line and hence collapses with no extension problems. Thus $H^{*}(F) \cong H^{*}(B G)$. By naturality of the BSS and the structure of $H^{*}(X)$ it follows at once that $F$ is comparable to $B G$. By hypothesis, $G$ is determined by cohomology, hence $F \simeq B G$ and therefore $X$ is the classifying space of a $p$-group $K$, given as an extension

$$
1 \rightarrow G \rightarrow K \rightarrow E \rightarrow 1
$$

Central elements of order $p$ in $K$ are cohomologically detectable by means of the Lannes' $T$ functor [13]. Since $H^{*}(B K) \cong H^{*}(B(G \times E))$ as algebras over the Steenrod algebra, the central elements of order $p$ in $K$ and $G \times E$ are in one to one correspondence. Hence $K$ contains a central subgroup isomorphic to $E$ and an inclusion $s$ can be chosen so that the induced map on cohomology is the obvious projection. Thus $s$ is a section for $\phi$ and the extension (7.1) is therefore split. Since the section $s$ is central, the action of $E$ on $G$ is trivial and so $K \cong G \times E$ and $X \simeq B K \simeq B(G \times E)$.

\section{REFERENCES}

[1] J. Aguadé, Cohomology algebras with two generators, Math. Z. 177 (1981), 289-296. MR 82d:55003

[2] J. Aguadé, C. Broto and D. Notbohm, Homotopy classification of spaces with interesting cohomology and a conjecture of Cooke, part I, Topology 33 (1994), 455-492. MR 95i:55006

[3] J. Aguadé, C. Broto and M. Santos, Fake three connected coverings of Lie groups, Duke Math. J. 80 (1995), 91-103. MR 96j:57050

[4] D. Benson and J. Carlson, Cohomology of extraspecial groups, Bull. London Math. Soc. 24 (1992), 209-235. MR 93b:20087

[5] A.K. Bousfield, Homological localization towers for groups and П-modules, Mem. Amer. Math. Soc. 186 (1977). MR 56:5688

[6] A.K. Bousfield and D. Kan, Localizations, completions and homotopy limits, Lecture Notes in Math. 304, Springer Verlag (1972). MR 51:1825

[7] W. Browder, Torsion in H-spaces Ann. Math. 74 (1961), 24-51. MR 23:A2201

[8] W. Dwyer, Strong convergence of the Eilenberg-Moore spectral sequence, Topology 13 (1973), 255-265. MR 52:15464

[9] L. Evens and S. B. Priddy, The cohomology of the semidihedral groups, Contemp. Math. 37 (1985), 61-72. MR 82h:20075

[10] Z. Fiedorowicz and S. B. Priddy, Homology of classical groups over finite fields and their associated infinite loop spaces, Lecture Notes in Math. 674, Springer Verlag (1978). MR 80g:55018

[11] R. Kane, The Homology of Hopf Spaces, North-Holland, (1988). MR 90f:55018

[12] I. Leary; 3-groups are not determined by their integral cohomology rings, Preprint.

[13] G. Mislin, Cohomologically central elements and fusion in groups, Algebraic Topology, Homotopy and Group Cohomology, Proceedings, Barcelona 1990, Lecture Notes in Math. 1509, Springer Verlag (1992). MR 94d:55032

[14] D. Quillen, The mod-2 cohomology rings of extra special 2-groups and the spinor groups, Math. Ann. 194 (1971), 197-212. MR 44:7582 
[15] D. J. Rusin, The mod-2 cohomology of metacyclic 2-groups, J. Pure Appl. Algebra 44 (1987), 315-327. MR 88k:20076

[16] D. J. Rusin, The cohomology of groups of order 32, Math. Comp. 53 (1989), 359-385. MR 89k:20078

Departament de Matemàtiques, Universitat Autònoma de Barcelona, E-08193 BelLATERRA, Spain

E-mail address: broto@mat.uab.es

Mathematisches Institut, Universität Heidelberg, INF 288, Heidelberg 69120, GerMANY

Current address: Department of Mathematics, Northwestern University, 2033 Sheridan Rd., Evanston, Illinois 60201

E-mail address: ran@math.nwu.edu 